\title{
A Discrete Chloride Monohydrate: a Solid State Structural and Spectroscopic Characterization
}

\author{
Mohammed S. Abdelbassit ${ }^{1}$, Owen J. Curnow ${ }^{1 *}$, Eugen Libowitzky², Ross O. Piltz ${ }^{3}$ and Matthew I. J. \\ Polson ${ }^{1}$ \\ ${ }^{1}$ School of Physical and Chemical Sciences, University of Canterbury, Private Bag 4800, \\ Christchurch 8140, New Zealand. 'Institut für Mineralogie und Kristallographie, Universität Wien, \\ Geozentrum, Althanstraße 14, A-1090 Vienna, Austria. ${ }^{3}$ Australian Centre for Neutron Scattering, \\ Lucas Heights, NSW 2234, Australia. *e-mail: owen.curnow@canterbury.ac.nz
}

\section{Supplementary Information}

\section{Experimental}

All the experimental work and sample preparation were carried out under dried nitrogen atmosphere using standard Schlenk techniques. $\mathrm{CH}_{2} \mathrm{Cl}_{2}$, diethylether, $\mathrm{D}_{2} \mathrm{O}$ and 1,2-dibromoethane were obtained commercially. Solvents were dried using an in-house solvent purification system. $\left[\mathrm{C}_{3}\left(\mathrm{NPr}_{2}\right)_{3}\right] \mathrm{Cl}$ was prepared as described in reference 1.

Far-IR data were collected at room temperature by using a Vertex 70 FT-IR spectrometer from Bruker (Germany), operating with a Platinum ATR unit with a diamond crystal. A resolution of $4 \mathrm{~cm}^{-1}$ and 16 scans were taken. The region from 680 to $30 \mathrm{~cm}^{-1}$ was covered by a DTGS detector. Melting point and decomposition profile were determined by coupled DSC/TGA using a TA Instruments SDT Q600 at $10^{\circ} \mathrm{C} / \mathrm{min}$. Microanalyses were performed by Campbell Microanalytical Laboratory, University of Otago, Dunedin.

Tris(dipropylamino)cyclopropenium chloride monohydrate $\left[\mathrm{C}_{3}\left(\mathrm{NPr}_{2}\right)_{3}\right] \mathrm{Cl}_{\mathbf{2}} \mathbf{H}_{2} \mathbf{O} \cdot\left[\mathrm{C}_{3}\left(\mathrm{NPr}_{2}\right)_{3}\right] \mathrm{Cl}(3.000 \mathrm{~g}$, $8.062 \mathrm{mmol}$ ) in liquid form was mixed with diethylether $(50 \mathrm{~mL})$. The mixture was kept in an open beaker to allow the ether to slowly evaporate and colourless hexagonal crystals $(2.602 \mathrm{~g}, 83 \%$ yield) formed overnight. $T_{\mathrm{m}}=67.5^{\circ} \mathrm{C}$. Elemental analysis calcd (\%) for $\mathrm{C}_{21} \mathrm{H}_{44} \mathrm{~N}_{3} \mathrm{ClO}: \mathrm{C}, 64.64 ; \mathrm{H}, 11.39 ; \mathrm{N}, 10.77$; Found: $\mathrm{C}, 64.76$; $\mathrm{H}, 11.03 ; \mathrm{N}, 10.67$.

$\left[\mathbf{C}_{3}\left(\mathrm{NPr}_{2}\right)_{3}\right] \mathbf{C l} . \mathbf{D}_{2} \mathrm{O}$ and $\left[\mathbf{C}_{3}\left(\mathrm{NPr}_{2}\right)_{3}\right] \mathbf{C l} . \mathrm{HDO} .\left[\mathrm{C}_{3}\left(\mathrm{NPr}_{2}\right)_{3}\right] \mathrm{Cl}(2.000 \mathrm{~g}, 5.375 \mathrm{mmol})$ was dissolved in dry dichloromethane $(30 \mathrm{~mL})$. $\mathrm{D}_{2} \mathrm{O}$ or a 1:1 mixture of $\mathrm{H}_{2} \mathrm{O}$ and $\mathrm{D}_{2} \mathrm{O}(0.3 \mathrm{~mL}, 15 \mathrm{mmol})$, which gives a 1:2:1 ratio of $\mathrm{H}_{2} \mathrm{O} / \mathrm{HDO} / \mathrm{D}_{2} \mathrm{O}$, was added and the dichloromethane was then removed in vacuo. Dry diethylether $(30 \mathrm{~mL})$ was added to the mixture under nitrogen and kept overnight at ambient temperature. The excess diethylether was then removed in vacuo to give colourless crystals of the desired product (1.7-1.8 g, 80-85\% yield).

\section{Micro-Raman spectroscopy}

Raman spectra were collected by using a Renishaw RM1000 confocal edge filter-based micro-Raman system. The $632.8 \mathrm{~nm}$ excitation line of a $\sim 20 \mathrm{~mW}$ HeNe laser was focused with a 50x/0.75 objective lens on the sample surface. The back-scattered radiation ( $180^{\circ}$ configuration) was analyzed with a 1200 lines $/ \mathrm{mm}$ grating monochromator. Raman intensities were collected for 300 seconds with a thermo-electrically cooled CCD array detector. The spectral resolution of the system ("apparatus function") was $3-4 \mathrm{~cm}^{-1}$, the wavenumber accuracy was $\pm 1 \mathrm{~cm}^{-1}$ (both calibrated with the Rayleigh line and the $520.5 \mathrm{~cm}^{-1}$ line of a Si standard). The confocal setup limited the spatial (lateral and depth) resolution to $2-3 \mu \mathrm{m}$. A spectral overview at room temperature (RT) was obtained from $\sim 20$ to $4000 \mathrm{~cm}^{-1}$ using the continuous grating scan mode. A Linkam FTIR 600 heating/cooling stage (glass window + long-distance objective 50x/0.55), liquid nitrogen 
pump, TP93 temperature controller) was employed for low-temperature (LT) spectra from 300 to $80 \mathrm{~K}$ at steps of $20 \mathrm{~K}$. To avoid extended acquisition times, only the range $3110-3730 \mathrm{~cm}^{-1}$ was sampled using the static grating mode. Instrument control and data acquisition were done with Grams/32 software (Galactic Ind. Corp.).

\section{FTIR micro-spectroscopy}

Micro-FTIR spectra of a thin layer of crushed crystals were measured in reflection/absorption technique (the silver block of the Linkam FTIR 600 stage - see above - acting as retro-reflector) on a Bruker Tensor 27 FTIR spectrometer connected to a Bruker Hyperion IR microscope. The system was equipped with a glo(w)bar MIR light source, a KBr beam splitter, a 15x/0.4 Cassegrain type condenser and objective, and a liquid nitrogen-cooled MCT detector. The field of measurement was constrained by a $50 \mu \mathrm{m}$ square aperture. Sample and background spectra were averaged from 128 scans at $4 \mathrm{~cm}^{-1}$ spectral resolution. RT spectra were acquired without lid/window from 550 to $4000 \mathrm{~cm}^{-1}$. For the series of $\mathrm{LT}$ spectra a $\mathrm{BaF}_{2}$ window in the closed lid of the Linkam FTIR600 stage limited the range to $700-4000 \mathrm{~cm}^{-1}$. Instrument control and data handling was performed with OPUS 5.5 software (Bruker Optik $\mathrm{GmbH}, 2005)$. The accuracy of the heatingcooling stage has been confirmed with $n$-heptane $\left(\mathrm{MP}-91^{\circ} \mathrm{C}\right)$ by repeated freezing/melting between -90 and $-92{ }^{\circ} \mathrm{C}$. Correct stage temperatures are further confirmed by the observation of unwanted ice crystallisation/melting at $\sim 0^{\circ} \mathrm{C}$ and by the lowest possible temperature of the stage at $-195^{\circ} \mathrm{C}$ (pulling LN2 with a temperature of $-196^{\circ} \mathrm{C}$ through the silver block of the stage).

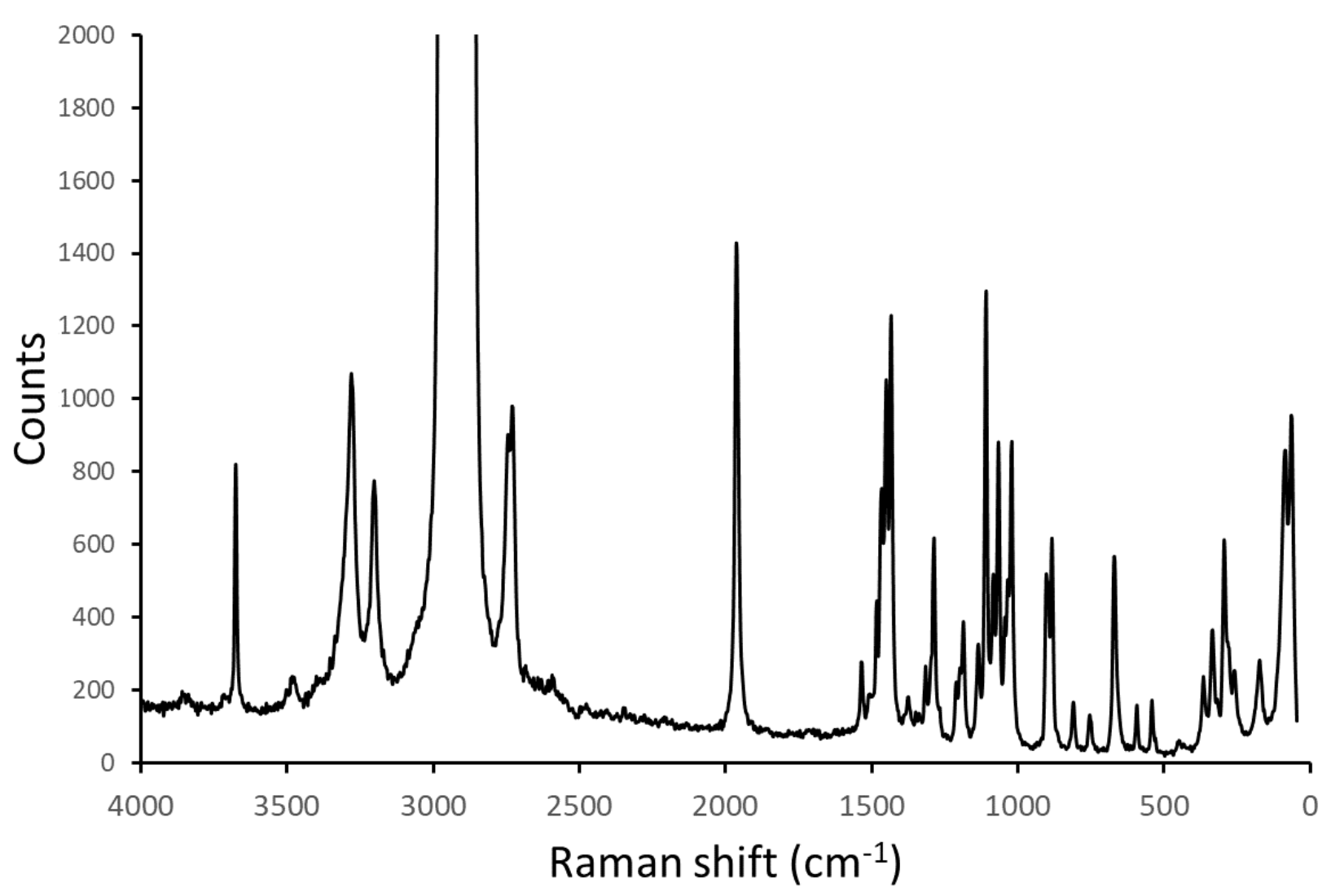

Figure 1S. Raman spectrum of $\left[\mathrm{C}_{3}\left(\mathrm{NPr}_{2}\right)_{3}\right] \mathrm{Cl} . \mathrm{H}_{2} \mathrm{O}$ at $300 \mathrm{~K}$. 


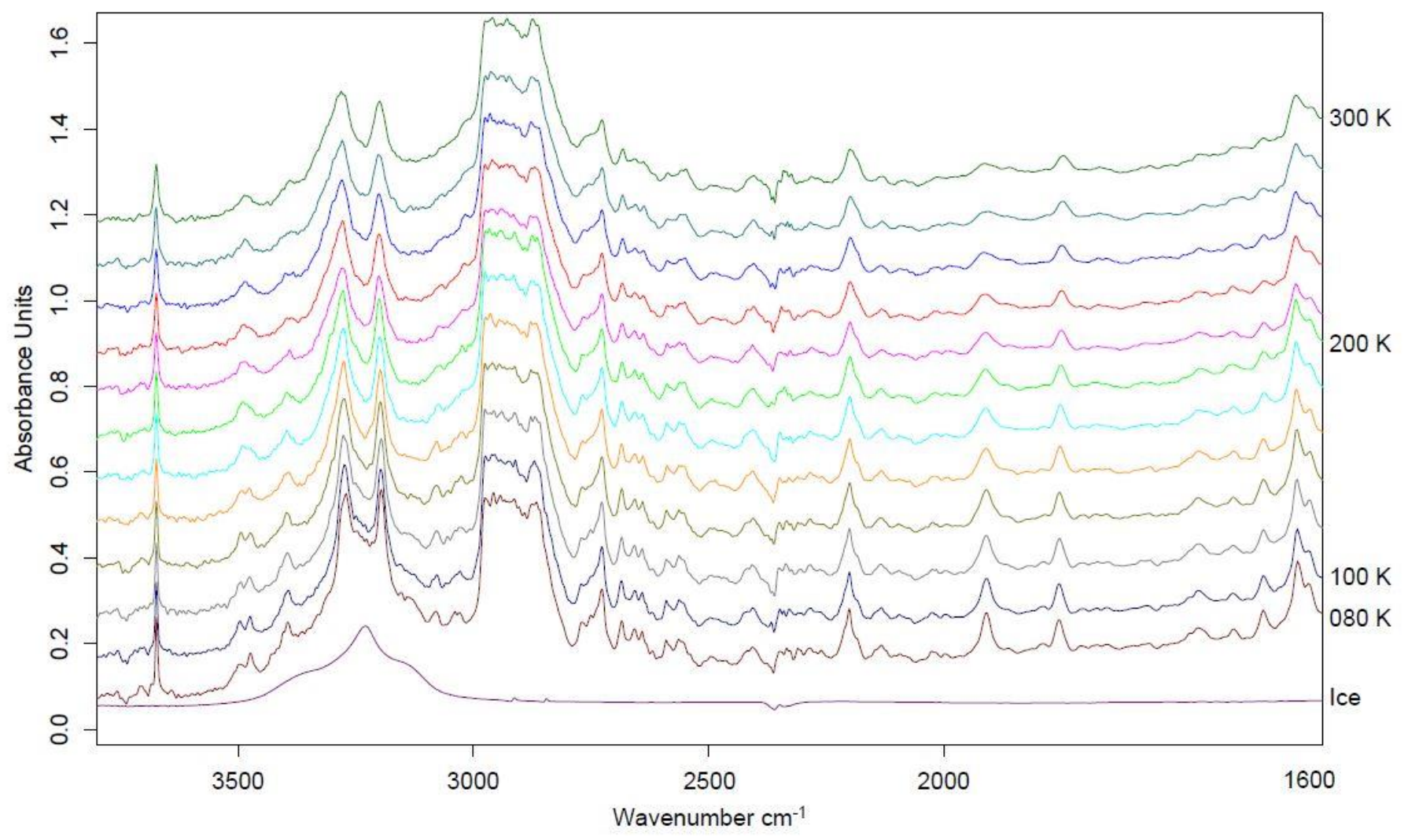

Figure 2S. Variable temperature infrared spectra of $\left[\mathrm{C}_{3}\left(\mathrm{NPr}_{2}\right)_{3}\right] \mathrm{Cl} . \mathrm{H}_{2} \mathrm{O}$ at $80-300 \mathrm{~K}$.

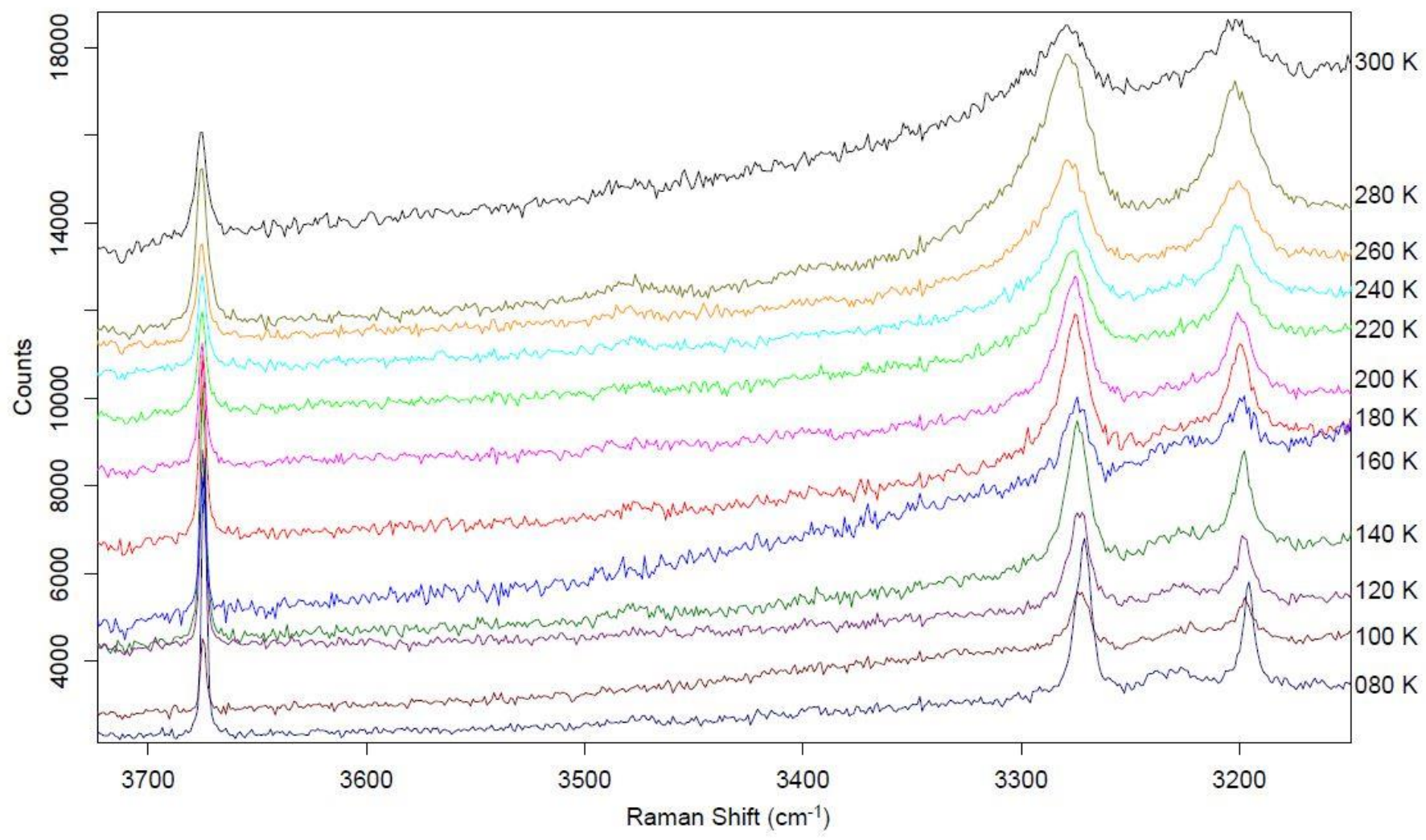

Figure 3S. Variable temperature Raman spectra of $\left[\mathrm{C}_{3}\left(\mathrm{NPr}_{2}\right)_{3}\right] \mathrm{Cl} . \mathrm{H}_{2} \mathrm{O}$ at $80-300 \mathrm{~K}$. 


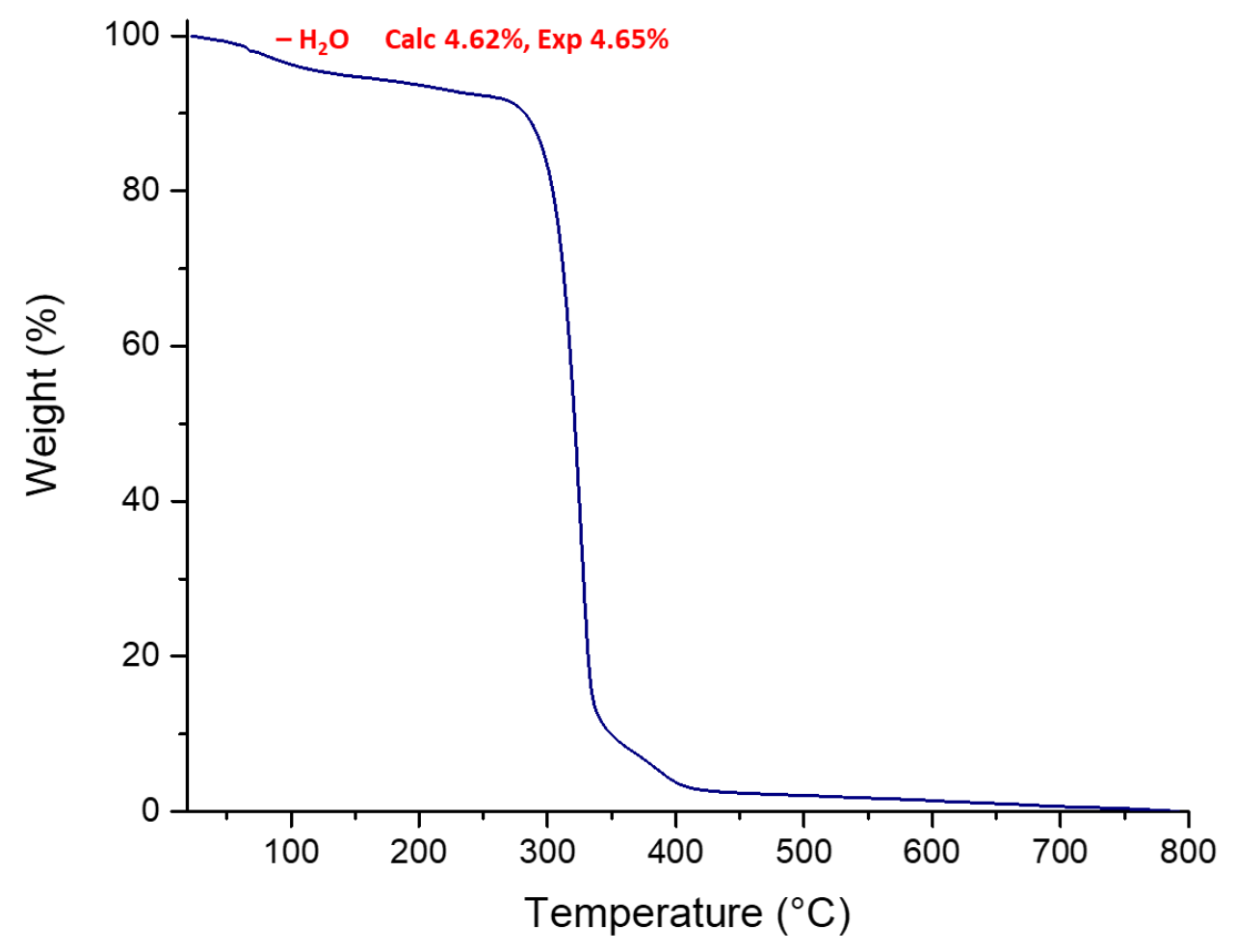

Figure 4S. Thermogravimetric analysis of $\left[\mathrm{C}_{3}\left(\mathrm{NPr}_{2}\right)_{3}\right] \mathrm{Cl}_{2} \mathrm{H}_{2} \mathrm{O}$.

\section{Crystal data}

To collect X-ray data, a suitable crystal was mounted on a SuperNova, Dual, Mo at zero, Atlas diffractometer. Data were collected and reduced using CrysAlisPro 1.171.38.43. Using Olex22 $2^{2}$ the structure was solved with the XT structure solution program ${ }^{3}$ using Intrinsic Phasing and refined with the XL refinement package $^{3}$ using Least Squares minimisation with anisotropic thermal parameters for all non-hydrogen atoms. Hydrogen atoms on the methylene groups were refined isotropically at their calculated positions and methyl groups were refined as rotating groups. The water protons were located from the density difference map and positionally-refined with riding thermal parameters. For the $120 \mathrm{~K}$ data, a small damp restraint (DAMP 20) was required to stop the model refining to having the oxygen on the rotational axis. Refinement with the oxygen on the axis had an unstable refinement of the thermal ellipsoids that never reached convergence, thus indicating that this position was an artefact associated with the symmetry element. At $120 \mathrm{~K}$, the water atoms were each refined with fixed site occupancy factors of 0.33333 as they refined best with all atoms off the $C_{3}$ axis. At $300 \mathrm{~K}$, the bridging $\mathrm{H}$ atom $\mathrm{H} 1 \mathrm{~A}$ refined as being on the $C_{3}$ axis, so only $\mathrm{O} 1$ and $\mathrm{H} 1 \mathrm{~B}$ were fixed at 00.33333 .

Neutron data were collected on KOALA at OPAL. KOALA uses 'white' thermal-neutron beams $(0.8 \AA \sim<$ $\sim<5.2 \AA$ ) with a cylindrical image-plate detector. The crystal was mounted inside the CF2 helium refrigerator and neutron Laue patterns were collected on KOALA in 25 exposures of $1 \mathrm{~h}$ each, separated by $8^{\circ}$ rotations of the $\phi$-axis (perpendicular to the neutron beam), providing good data redundancy. The image data was processed using the LaueG software ${ }^{4}$ to create hkl-intensity files using the methods described in References 5 and 6. Structural refinements were undertaken with the Jana2006 package. ${ }^{7}$ At both 120 and $300 \mathrm{~K}$, the bridging $\mathrm{H}$ atom $\mathrm{HB}$ refined as being on the $C_{3}$ axis, so only $\mathrm{O} 1$ and $\mathrm{HF}$ were fixed with 00.33333 site occupancy factors. 
Table 1S | Structure refinement data for $\left[\mathrm{C}_{3}\left(\mathrm{NPr}_{2}\right)_{3}\right] \mathrm{Cl} . \mathrm{H}_{2} \mathrm{O}$.

\begin{tabular}{|c|c|c|c|c|}
\hline & X-ray (Mo) $120 \mathrm{~K}$ & X-ray (Mo) $300 \mathrm{~K}$ & Neutron $120 \mathrm{~K}$ & Neutron $300 \mathrm{~K}$ \\
\hline formula & $\mathrm{C}_{21} \mathrm{H}_{44} \mathrm{ClN}_{3} \mathrm{O}$ & $\mathrm{C}_{21} \mathrm{H}_{44} \mathrm{CIN}_{3} \mathrm{O}$ & $\mathrm{C}_{21} \mathrm{H}_{44} \mathrm{CIN}_{3} \mathrm{O}$ & $\mathrm{C}_{21} \mathrm{H}_{44} \mathrm{ClN}_{3} \mathrm{O}$ \\
\hline$a[\AA]$ & $11.1075(3)$ & $11.1853(9)$ & $11.1004(2)$ & $11.1746(3)$ \\
\hline$b[\AA]$ & $11.1075(3)$ & 11.1853(9) & $11.1004(2)$ & $11.1746(3)$ \\
\hline$c[\AA]$ & $33.5179(13)$ & $34.533(3)$ & $33.4883(7)$ & $34.6593(12)$ \\
\hline$\alpha\left[^{\circ}\right]$ & 90 & 90 & 90 & 90 \\
\hline$\beta\left[^{\circ}\right]$ & 90 & 90 & 90 & 90 \\
\hline$V\left[^{\circ}\right]$ & 120 & 120 & 120 & 120 \\
\hline$V\left[\AA^{3}\right]$ & $3581.3(2)$ & $3741.6(7)$ & $3573.56(15)$ & $3748.1(2)$ \\
\hline$z$ & 6 & 6 & 6 & 6 \\
\hline$\rho_{\text {calc }}\left[\mathrm{g} \mathrm{cm}^{-3}\right]$ & 1.085 & 1.039 & 1.087 & 1.042 \\
\hline crystal system & trigonal & trigonal & trigonal & trigonal \\
\hline space group & $R-3$ & $R-3$ & $R-3$ & $R-3$ \\
\hline shape/colour & colourless & colourless & colourless & colourless \\
\hline crystal size [mm] & $0.33 \times 0.25 \times 0.10$ & $0.33 \times 0.25 \times 0.10$ & $1.3 \times 1.2 \times 1.0$ & $1.3 \times 1.2 \times 1.0$ \\
\hline$\mu\left[\mathrm{mm}^{-1}\right]$ & 0.174 & 0.166 & 0.075 & 0.071 \\
\hline$T[\mathrm{~K}]$ & $120.0(1)$ & $300.0(1)$ & 120 & 293(2) \\
\hline$F(000)$ & 1296 & 1296 & 1296 & 1296 \\
\hline $2 \theta$ range $\left[{ }^{\circ}\right]$ & 7.294 to 75.388 & 7.08 to 59.738 & $5.905-141.31$ & $7.718-136.576$ \\
\hline \multirow[t]{3}{*}{ index ranges } & $-14 \leq h \leq 15$ & $-13 \leq h \leq 14$ & $-20 \leq h \leq 20$ & $-15 \leq h \leq 15$ \\
\hline & $-18 \leq k \leq 16$ & $-10 \leq k \leq 15$ & $-16 \leq k \leq 20$ & $-16 \leq k \leq 7$ \\
\hline & $-56 \leq I \leq 57$ & $-48 \leq I \leq 41$ & $-50 \leq I \leq 51$ & $-52 \leq 1 \leq 52$ \\
\hline reflections collected & 10919 & 3798 & 53379 & 24249 \\
\hline independent reflns & 4043 & 1974 & 3734 & 2498 \\
\hline$R$ (int) & 0.0215 & 0.0208 & 0.7179 & 1.1273 \\
\hline data/restraints/parameters & $4043 / 2 / 93$ & $1974 / 3 / 91$ & $3734 / 0 / 223$ & $2498 / 0 / 223$ \\
\hline GoF on $F^{2}$ & 1.041 & 1.025 & 1.188 & 0.991 \\
\hline$R_{1} / w R_{2}[l>2 \sigma(I)]$ & $0.0385 / 0.0978$ & $0.0406 / 0.1037$ & $0.1479 / 0.2226$ & $0.0956 / 0.0864$ \\
\hline$R_{1} / w R_{2}$ (all data) & $0.0504 / 0.1050$ & $0.0786 / 0.1149$ & $0.3100 / 0.2740$ & $0.3169 / 0.1162$ \\
\hline$\Delta \rho_{\max / \min } / \mathrm{e}\left[\AA^{-3}\right]$ & $0.43 /-0.27$ & $0.12 /-0.16$ & $1.56 /-1.50$ & $0.45 /-0.37$ \\
\hline CCDC number & 1968612 & 1968613 & 1968701 & 1968699 \\
\hline
\end{tabular}


Table 2S | Distances $(\AA)$ and angles $\left({ }^{\circ}\right)$ for X-ray diffraction study at $120 \mathrm{~K}$.

\begin{tabular}{|c|c|c|c|}
\hline $\mathrm{C} 1-\mathrm{C} 1$ & $1.3869(10)$ & $\mathrm{N} 1-\mathrm{C} 1$ & $1.3288(8)$ \\
\hline N1-C11 & $1.4688(8)$ & N1-C14 & $1.4656(8)$ \\
\hline C11-C12 & $1.5193(10)$ & C14-C15 & $1.5184(10)$ \\
\hline C12-C13 & $1.5231(10)$ & C15-C16 & $1.5207(11)$ \\
\hline $\mathrm{O} 1-\mathrm{H} 1 \mathrm{~A}$ & $0.88(3)$ & O1-H1B & $0.87(4)$ \\
\hline C1-C1-C1 & 60.000 & N1-C1-C1 & $150.26(7)$ \\
\hline C11-N1-C14 & $118.77(5)$ & N1-C1-C1 & $149.74(7)$ \\
\hline C1-N1-C11 & $119.43(5)$ & C1-N1-C14 & $120.34(5)$ \\
\hline N1-C11-C12 & $112.05(5)$ & N1-C14-C15 & $112.05(6)$ \\
\hline C13-C12-C11 & $111.87(6)$ & C14-C15-C16 & 111.95(6) \\
\hline $\mathrm{Hf}-\mathrm{O} 1-\mathrm{Hb}$ & $119(13)$ & & \\
\hline O1---C $C_{3}$ plane & $3.337(2)$ & & \\
\hline Cl1---O1 & $3.110(2)$ & Cl1---Hb & $2.24(3)$ \\
\hline $\mathrm{Cl} 1-\mathrm{Hb}-\mathrm{O} 1$ & $168(20)$ & & \\
\hline C14---Cl1 & $3.7228(9)$ & C11---Cl1 & $3.8279(9)$ \\
\hline C13---Cl1 & $4.0399(9)$ & & \\
\hline
\end{tabular}

Table 3S | Distances $(\AA ̊)$ and angles $\left({ }^{\circ}\right)$ for X-ray diffraction study at $300 \mathrm{~K}$.

\begin{tabular}{|c|c|c|c|}
\hline $\mathrm{C} 1-\mathrm{C} 1$ & $1.381(2)$ & N1-C1 & $1.3285(17)$ \\
\hline N1-C11 & $1.4665(18)$ & N1-C14 & $1.4596(18)$ \\
\hline C11-C12 & $1.496(2)$ & C14-C15 & $1.507(2)$ \\
\hline C12-C13 & $1.514(2)$ & C15-C16 & $1.504(3)$ \\
\hline $\mathrm{O} 1-\mathrm{H} 1 \mathrm{~A}$ & $0.89(2)$ & O1-H1B & $0.87(6)$ \\
\hline C1-C1-C1 & $59.999(1)$ & $\mathrm{N} 1-\mathrm{C} 1-\mathrm{C} 1$ & $149.96(16)$ \\
\hline C14-N1-C11 & $118.66(11)$ & N1-C1-C1 & $150.04(16)$ \\
\hline C1-N1-C11 & 119.72(11) & C1-N1-C14 & $120.47(12)$ \\
\hline N1-C11-C12 & $112.92(12)$ & N1-C14-C15 & $112.71(13)$ \\
\hline C11-C12-C13 & $112.95(13)$ & C16-C15-C14 & $112.74(15)$ \\
\hline $\mathrm{Hb}-\mathrm{O} 1-\mathrm{Hf}$ & $102(4)$ & & \\
\hline O1--- $C_{3}$ plane & $3.496(5)$ & & \\
\hline Cl1---O1 & $3.151(5)$ & Cl1---Hb & $2.28(2)$ \\
\hline $\mathrm{Cl} 1-\mathrm{Hb}-\mathrm{O} 1$ & $167(2)$ & & \\
\hline C14---Cl1 & $3.7547(13)$ & C11---Cl1 & $3.8867(18)$ \\
\hline C13---Cl1 & $4.129(2)$ & & \\
\hline
\end{tabular}

Table 4S | Distances $(\AA ̊)$ and angles $\left({ }^{\circ}\right)$ for neutron diffraction study at $120 \mathrm{~K}$.

\begin{tabular}{llll}
\hline C1-C1 & $1.384(4)$ & $\mathrm{N} 1-\mathrm{C} 1$ & $1.329(3)$ \\
$\mathrm{N} 1-\mathrm{C} 5$ & $1.467(3)$ & $\mathrm{N} 1-\mathrm{C} 2$ & $1.465(3)$ \\
$\mathrm{C} 5-\mathrm{C} 6$ & $1.524(5)$ & $\mathrm{C} 2-\mathrm{C} 3$ & $1.526(4)$ \\
$\mathrm{C} 6-\mathrm{C} 7$ & $1.517(4)$ & $\mathrm{C} 3-\mathrm{C} 4$ & $1.523(4)$ \\
$\mathrm{C} 5-\mathrm{H} 5 \mathrm{~A}$ & $1.085(6)$ & $\mathrm{C} 2-\mathrm{H} 2 \mathrm{~B}$ & $1.099(7)$ \\
$\mathrm{C} 5-\mathrm{H} 5 \mathrm{~B}$ & $1.092(6)$ & $\mathrm{C} 2-\mathrm{H} 2 \mathrm{~A}$ & $1.099(6)$ \\
$\mathrm{C} 6-\mathrm{H} 6 \mathrm{~A}$ & $1.086(8)$ & $\mathrm{C} 3-\mathrm{H} 3 \mathrm{~A}$ & $1.094(8)$ \\
$\mathrm{C} 6-\mathrm{H} 6 \mathrm{~B}$ & $1.090(9)$ & $\mathrm{C} 3-\mathrm{H} 3 \mathrm{~B}$ & $1.097(7)$ \\
$\mathrm{C} 7-\mathrm{H} 7 \mathrm{~A}$ & $1.096(12)$ & $\mathrm{C} 4-\mathrm{H} 4 \mathrm{~A}$ & $1.090(9)$
\end{tabular}




\begin{tabular}{|c|c|c|c|}
\hline $\mathrm{C} 7-\mathrm{H} 7 \mathrm{~B}$ & $1.076(11)$ & $\mathrm{C} 4-\mathrm{H} 4 \mathrm{~B}$ & $1.082(9)$ \\
\hline $\mathrm{C} 7-\mathrm{H} 7 \mathrm{C}$ & $1.086(9)$ & $\mathrm{C} 4-\mathrm{H} 4 \mathrm{C}$ & $1.086(8)$ \\
\hline $\mathrm{O} 1-\mathrm{Hb}$ & $0.93(5)$ & $\mathrm{O} 1-\mathrm{Hf}$ & $0.75(5)$ \\
\hline C1-C1-C1 & $60.000(1)$ & N1-C1-C1 & $150.3(3)$ \\
\hline C2-N1-C5 & $118.60(18)$ & $\mathrm{N} 1-\mathrm{C} 1-\mathrm{C} 1$ & $149.7(3)$ \\
\hline C1-N1-C5 & $120.32(18)$ & C1-N1-C2 & $119.67(18)$ \\
\hline N1-C5-C6 & $111.8(2)$ & $\mathrm{N} 1-\mathrm{C} 2-\mathrm{C} 3$ & $112.1(2)$ \\
\hline $\mathrm{H} 5 \mathrm{~A}-\mathrm{C} 5-\mathrm{N} 1$ & $108.1(4)$ & $\mathrm{H} 2 \mathrm{~B}-\mathrm{C} 2-\mathrm{N} 1$ & $109.6(4)$ \\
\hline H5A-C5-C6 & $110.6(5)$ & $\mathrm{H} 2 \mathrm{~B}-\mathrm{C} 2-\mathrm{C} 3$ & $110.0(5)$ \\
\hline H5A-C5-H5B & $109.0(6)$ & $\mathrm{H} 2 \mathrm{~B}-\mathrm{C} 2-\mathrm{H} 2 \mathrm{~A}$ & $108.1(7)$ \\
\hline H5B-C5-N1 & $107.6(4)$ & $\mathrm{H} 2 \mathrm{~A}-\mathrm{C} 2-\mathrm{N} 1$ & $107.4(4)$ \\
\hline $\mathrm{H} 5 \mathrm{~B}-\mathrm{C} 5-\mathrm{C} 6$ & $109.7(5)$ & $\mathrm{H} 2 \mathrm{~A}-\mathrm{C} 2-\mathrm{C} 3$ & $109.6(5)$ \\
\hline C7-C6-C5 & $111.9(3)$ & $\mathrm{C} 4-\mathrm{C} 3-\mathrm{C} 2$ & $111.9(3)$ \\
\hline $\mathrm{H} 6 \mathrm{~A}-\mathrm{C} 6-\mathrm{C} 5$ & $108.9(5)$ & $\mathrm{H} 3 \mathrm{~A}-\mathrm{C} 3-\mathrm{C} 2$ & $107.4(5)$ \\
\hline H6A-C6-C7 & $110.9(5)$ & $\mathrm{H} 3 \mathrm{~A}-\mathrm{C} 3-\mathrm{C} 4$ & $110.7(4)$ \\
\hline H6A-C6-H6B & $107.3(8)$ & H3A-С3-H3B & $107.0(7)$ \\
\hline H6B-C6-C5 & $108.1(6)$ & H3B-C3-C2 & $109.3(5)$ \\
\hline $\mathrm{H} 6 \mathrm{~B}-\mathrm{C} 6-\mathrm{C} 7$ & $109.6(6)$ & H3B-C3-C4 & $110.4(4)$ \\
\hline H7A-C7-C6 & $111.3(6)$ & $\mathrm{H} 4 \mathrm{~A}-\mathrm{C} 4-\mathrm{C} 3$ & $111.9(5)$ \\
\hline H7B-C7-C6 & $112.0(7)$ & $\mathrm{H} 4 \mathrm{~B}-\mathrm{C} 4-\mathrm{C} 3$ & $111.1(5)$ \\
\hline H7B-C7-H7A & $107.0(10)$ & $\mathrm{H} 4 \mathrm{~B}-\mathrm{C} 4-\mathrm{H} 4 \mathrm{~A}$ & $107.5(8)$ \\
\hline H7B-C7-H7C & 108.6(9) & $\mathrm{H} 4 \mathrm{~B}-\mathrm{C} 4-\mathrm{H} 4 \mathrm{C}$ & 107.3(8) \\
\hline H7C-C7-C6 & $111.0(6)$ & $\mathrm{H} 4 \mathrm{C}-\mathrm{C} 4-\mathrm{C} 3$ & $110.6(5)$ \\
\hline H7C-C7-H7A & $106.8(10)$ & $\mathrm{H} 4 \mathrm{C}-\mathrm{C} 4-\mathrm{H} 4 \mathrm{~A}$ & $108.2(8)$ \\
\hline $\mathrm{Hf}-\mathrm{O} 1-\mathrm{Hb}$ & $102(4)$ & & \\
\hline O1---Cl1 & $3.09(2)$ & $\mathrm{Cl} 1---\mathrm{Hb}$ & $2.16(4)$ \\
\hline $\mathrm{Cl} 1-\mathrm{HB}-\mathrm{O} 1$ & $175(6)$ & $\mathrm{Hf}-\mathrm{O} 1-\mathrm{Hb}-\mathrm{Cl} 1$ & $152(51)$ \\
\hline C2---Cl1 & $3.819(2)$ & $\mathrm{Cl} 1-\mathrm{H} 2 \mathrm{~A}$ & $2.820(7)$ \\
\hline $\mathrm{C} 2-\mathrm{H} 2 \mathrm{~A}-\mathrm{Cl} 1$ & $151.1(7)$ & & \\
\hline C5---Cl1 & $3.7199(17)$ & $\mathrm{Cl} 1-\mathrm{H} 5 \mathrm{~B}$ & $2.750(5)$ \\
\hline $\mathrm{C} 5-\mathrm{H} 5 \mathrm{~B}-\mathrm{Cl} 1$ & $147.9(5)$ & & \\
\hline C4---Cl1 & $4.034(5)$ & $\mathrm{Cl} 1-\mathrm{H} 4 \mathrm{~A}$ & $2.957(11)$ \\
\hline $\mathrm{C} 4-\mathrm{H} 4 \mathrm{~A}-\mathrm{Cl} 1$ & $170.0(9)$ & & \\
\hline
\end{tabular}

Table 5S | Distances $(\AA)$ and angles $\left({ }^{\circ}\right)$ for neutron diffraction study at $300 \mathrm{~K}$.

\begin{tabular}{llll}
\hline $\mathrm{C} 1-\mathrm{C} 1$ & $1.386(3)$ & $\mathrm{N} 1-\mathrm{C} 1$ & $1.326(2)$ \\
$\mathrm{N} 1-\mathrm{C} 2$ & $1.460(3)$ & $\mathrm{N} 1-\mathrm{C} 5$ & $1.467(3)$ \\
$\mathrm{C} 2-\mathrm{C} 3$ & $1.511(4)$ & $\mathrm{C}-\mathrm{C} 6$ & $1.512(4)$ \\
$\mathrm{C} 3-\mathrm{C} 4$ & $1.515(5)$ & $\mathrm{C} 6-\mathrm{C} 7$ & $1.514(3)$ \\
$\mathrm{C} 2-\mathrm{H} 2 \mathrm{~A}$ & $1.091(6)$ & $\mathrm{C}-\mathrm{H} 5 \mathrm{~A}$ & $1.095(7)$ \\
$\mathrm{C} 2-\mathrm{H} 2 \mathrm{~B}$ & $1.083(6)$ & $\mathrm{C} 5-\mathrm{H} 5 \mathrm{~B}$ & $1.093(6)$ \\
$\mathrm{C} 3-\mathrm{H} 3 \mathrm{~A}$ & $1.101(9)$ & $\mathrm{C}-\mathrm{H} 6 \mathrm{~A}$ & $1.076(8)$ \\
$\mathrm{C} 3-\mathrm{H} 3 \mathrm{~B}$ & $1.091(12)$ & $\mathrm{C} 6-\mathrm{H} 6 \mathrm{~B}$ & $1.077(8)$ \\
$\mathrm{C} 4-\mathrm{H} 4 \mathrm{~A}$ & $1.031(16)$ & $\mathrm{C}-\mathrm{H} 7 \mathrm{~A}$ & $1.076(10)$ \\
$\mathrm{C} 4-\mathrm{H} 4 \mathrm{~B}$ & $1.029(14)$ & $\mathrm{C} 7-\mathrm{H} 7 \mathrm{~B}$ & $1.055(10)$ \\
$\mathrm{C} 4-\mathrm{H} 4 \mathrm{C}$ & $1.056(13)$ & $\mathrm{C}-\mathrm{H} 7 \mathrm{C}$ & $1.075(10)$ \\
$\mathrm{O} 1-\mathrm{Hb}$ & $0.87(4)$ & $\mathrm{O} 1-\mathrm{Hf}$ & $0.95(9)$
\end{tabular}




\begin{tabular}{|c|c|c|c|}
\hline C1-C1-C1 & $60.000(1)$ & $\mathrm{N} 1-\mathrm{C} 1-\mathrm{C} 1$ & $149.8(2)$ \\
\hline C2-N1-C5 & $118.61(18)$ & $\mathrm{N} 1-\mathrm{C} 1-\mathrm{C} 1$ & $150.2(2)$ \\
\hline C1-N1-C2 & $120.56(16)$ & C1-N1-C5 & $119.67(17)$ \\
\hline $\mathrm{N} 1-\mathrm{C} 2-\mathrm{C} 3$ & $112.54(19)$ & N1-C5-C6 & $112.5(2)$ \\
\hline $\mathrm{H} 2 \mathrm{~A}-\mathrm{C} 2-\mathrm{N} 1$ & 107.9(3) & H5A-C5-N1 & $109.4(3)$ \\
\hline $\mathrm{H} 2 \mathrm{~A}-\mathrm{C} 2-\mathrm{C} 3$ & $111.2(4)$ & H5A-C5-C6 & $110.3(3)$ \\
\hline $\mathrm{H} 2 \mathrm{~B}-\mathrm{C} 2-\mathrm{N} 1$ & $107.6(3)$ & H5B-C5-N1 & $107.3(3)$ \\
\hline $\mathrm{H} 2 \mathrm{~B}-\mathrm{C} 2-\mathrm{C} 3$ & $109.9(3)$ & H5B-C5-C6 & $109.6(4)$ \\
\hline $\mathrm{H} 2 \mathrm{~B}-\mathrm{C} 2-\mathrm{H} 2 \mathrm{~A}$ & $107.5(4)$ & H5B-C5-H5A & $107.5(5)$ \\
\hline C2-C3-C4 & $112.1(3)$ & C5-C6-C7 & $112.1(3)$ \\
\hline $\mathrm{H} 3 \mathrm{~A}-\mathrm{C} 3-\mathrm{C} 2$ & $108.0(4)$ & $\mathrm{H} 6 \mathrm{~A}-\mathrm{C} 6-\mathrm{C} 5$ & $109.1(4)$ \\
\hline $\mathrm{H} 3 \mathrm{~A}-\mathrm{C} 3-\mathrm{C} 4$ & $110.2(5)$ & $\mathrm{H} 6 \mathrm{~A}-\mathrm{C} 6-\mathrm{C} 7$ & $111.1(4)$ \\
\hline $\mathrm{H} 3 \mathrm{~B}-\mathrm{C} 3-\mathrm{C} 2$ & $107.5(5)$ & $\mathrm{H} 6 \mathrm{~A}-\mathrm{C} 6-\mathrm{H} 6 \mathrm{~B}$ & $106.1(5)$ \\
\hline H3B-C3-C4 & $110.7(5)$ & H6B-C6-C5 & $108.6(4)$ \\
\hline H3B-C3-H3A & $108.2(7)$ & $\mathrm{H} 6 \mathrm{~B}-\mathrm{C} 6-\mathrm{C} 7$ & $109.5(4)$ \\
\hline $\mathrm{H} 4 \mathrm{~A}-\mathrm{C} 4-\mathrm{C} 3$ & $112.1(6)$ & H7A-C7-C6 & $113.0(4)$ \\
\hline $\mathrm{H} 4 \mathrm{~A}-\mathrm{C} 4-\mathrm{H} 4 \mathrm{C}$ & $107.8(9)$ & H7B-C7-C6 & $111.4(4)$ \\
\hline H4B-C4-C3 & $111.1(6)$ & H7B-C7-H7A & $107.9(6)$ \\
\hline H4B-C4-H4A & $106.3(9)$ & H7B-C7-H7C & $107.1(6)$ \\
\hline H4B-C4-H4C & $108.4(8)$ & $\mathrm{H} 7 \mathrm{C}-\mathrm{C} 7-\mathrm{C} 6$ & $111.0(4)$ \\
\hline $\mathrm{H} 4 \mathrm{C}-\mathrm{C} 4-\mathrm{C} 3$ & $110.9(7)$ & $\mathrm{H} 7 \mathrm{C}-\mathrm{C} 7-\mathrm{H} 7 \mathrm{~A}$ & $106.1(6)$ \\
\hline $\mathrm{Hb}-\mathrm{O} 1-\mathrm{Hf}$ & $93(7)$ & & \\
\hline Cl1---O1 & $3.06(4)$ & $\mathrm{Cl} 1---\mathrm{Hb}$ & $2.21(2)$ \\
\hline $\mathrm{Cl} 1-\mathrm{Hb}-\mathrm{O} 1$ & $167(4)$ & $\mathrm{Cl} 1-\mathrm{Hb}-01-\mathrm{Hf}$ & $150(21)$ \\
\hline C5---Cl1 & $3.878(4)$ & $\mathrm{Cl} 1-\mathrm{H} 5 \mathrm{~B}$ & $2.898(7)$ \\
\hline $\mathrm{C} 5-\mathrm{H} 5 \mathrm{~B}-\mathrm{Cl} 1$ & $149.3(5)$ & & \\
\hline C2---Cl1 & $3.750(3)$ & $\mathrm{Cl} 1-\mathrm{H} 2 \mathrm{~B}$ & $2.769(7)$ \\
\hline $\mathrm{C} 2-\mathrm{H} 2 \mathrm{~B}-\mathrm{Cl} 1$ & $150.6(4)$ & & \\
\hline C7---Cl01 & $4.138(5)$ & $\mathrm{Cl} 1-\mathrm{H} 7 \mathrm{~A}$ & $3.076(7))$ \\
\hline C7-H7A-Cl1 & $169.5(5)$ & & \\
\hline
\end{tabular}

\section{References}

1. Curnow, O. J.; MacFarlane, D. R.; Walst, K. J. Triaminocyclopropenium salts as ionic liquids. Chem. Commun. 2011, 47, 10248-10250.

2. Dolomanov, O. V.; Bourhis, L. J.; Gildea, R. J.; Howard, J. A. K.; Puschmann, H. OLEX2: a complete structure solution, refinement and analysis program. J. Appl. Cryst. 2009, 42, 339-341.

3. Sheldrick, G. M. A short history of SHELX. Acta Cryst. A 2008, 64, 112-122.

4. Piltz, R. O. LaueG software for displaying and processing neutron Laue images. J. Appl. Crystallogr. 2018, 51, 963-965.

5. Wilkinson, C.; Khamis, H. W.; Stansfield, R. F. D.; McIntyre, G. J. Integration of single-crystal reflections using area multidetectors. J. Appl. Cryst. 1988, 21, 471-478.

6. Piltz, R. O. Accurate data processing for neutron Laue diffractometers. J. Appl. Crystallogr. 2018, 51, 635-645.

7. Petricek, V.; Dusek, M.; Palatinus, L., JANA2006 The crystallographic computing system, 2007 Institute of Physics, Praha, Czech Republic. 